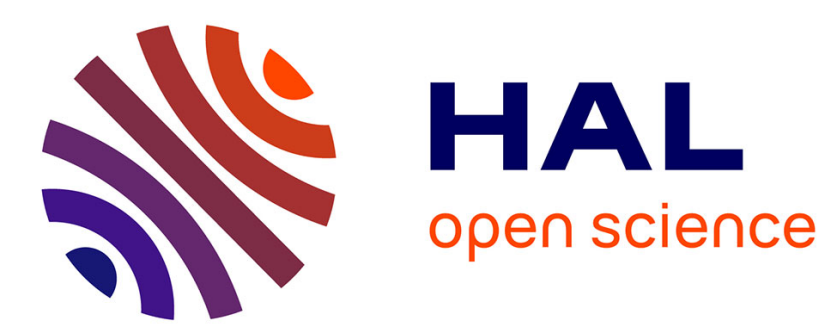

\title{
Simulation of Tremor on 3-Dimentional Musculoskeletal Model of Wrist Joint and Experimental Verification
}

\author{
Peng Yao, Dingguo Zhang, Mitsuhiro Hayashibe
}

\section{To cite this version:}

Peng Yao, Dingguo Zhang, Mitsuhiro Hayashibe. Simulation of Tremor on 3-Dimentional Musculoskeletal Model of Wrist Joint and Experimental Verification. EMBC 2012 - 34th International Conference of Engineering in Medicine and Biology, Aug 2012, San Diego, United States. pp.4823-4826, 10.1109/EMBC.2012.6347073 . lirmm-00740845

\section{HAL Id: lirmm-00740845 \\ https://hal-lirmm.ccsd.cnrs.fr/lirmm-00740845}

Submitted on 11 Oct 2012

HAL is a multi-disciplinary open access archive for the deposit and dissemination of scientific research documents, whether they are published or not. The documents may come from teaching and research institutions in France or abroad, or from public or private research centers.
L'archive ouverte pluridisciplinaire HAL, est destinée au dépôt et à la diffusion de documents scientifiques de niveau recherche, publiés ou non, émanant des établissements d'enseignement et de recherche français ou étrangers, des laboratoires publics ou privés. 


\title{
Simulation of Tremor on 3-Dimentional Musculoskeletal Model of Wrist Joint and Experimental Verification *
}

\author{
Peng Yao, Dingguo Zhang, Member, IEEE, Mitsuhiro Hayashibe, Member, IEEE
}

\begin{abstract}
A musculoskeletal model that allows to simulate the tremor of wrist joint with three degrees of freedom (DoFs) is developed. The model includes five muscles, extensor carpi radialis brevis, extensor carpi radialis longus, extensor carpi ulnaris, flexor carpi radialis and flexor carpi ulnaris. Simulation of tremor generation based on the 3-DoF model is performed. The tremor disorder can be generated in two directions: flexion-extension and radia-ulnar deviation. Accordingly, experiment is conducted on healthy subjects to verify the feasibility of artificial tremor generation via functional electrical stimulation (FES). Simulation results have shown qualitative agreement with the experimental results.
\end{abstract}

\section{INTRODUCTION}

Tremor can be broadly defined as any involuntary, approximately rhythmic and roughly sinusoidal movements of body parts in human [1]. A number of patients especially the elders are suffering from pathological tremor in the world. Musculoskeletal model plays an important role in study of tremor disorder. It can explore the inner mechanism or origin of tremor [2,3]; it can also be used as a controlled plant in simulation environment for assistive tremor suppression techniques $[4,5]$. However, the previous research is mostly accomplished based on a simple model with single degree-of-freedom (DoF) [2-7], which can not represent the real tremor in 3-dimensional space and brings bottleneck for further investigation. We are inspired to develop a complex musculoskeletal model with multiple DoFs and multiple muscles. As a case study, wrist joint is considered since it is one of the mostly related joints in the tremulous limbs of patients. In order to evaluate the performance of the model, experimental work based on functional electrical stimulation (FES) is conducted on healthy subjects. FES is a well-recognized technique in rehabilitation engineering that uses electrical pulses of low level to make skeletal muscles contract and generate desired movements. Here FES is adopted to make the subjects generate artificial tremor involuntarily.

\section{MusculoskeletAl Modeling}

Wrist is one of the most flexible joints in human, and wrist motion is essential for upper limb function. There are at least five main muscles and two skeletons in charge of

\footnotetext{
* Research supported by National Natural Science Foundation of China (No.51075265), and State Key Laboratory of Mechanical System and Vibration (No.MSVMS201112).

Peng Yao is with the School of Mechanical Engineering, Shanghai Jiao Tong University, China (e-mail: mepyao@foxmail.com).

Dingguo Zhang is with the School of Mechanical Engineering, Shanghai Jiao Tong University, China (corresponding author to provide phone: 86-021-34206072; fax: 86-021-34206072; e-mail: dgzhang@sjtu.edu.cn).

Mitsuhiro Hayashibe is with INRIA DEMAR Project and LIRMM, CNRS/University of Montpellier, France. (e-mail: hayashibe@lirmm.fr).
}

the wrist's movements in 3-dimentional space. The involved muscles are extensor carpi radialis brevis (ECRB), extensor carpi radialis longus (ECRL), extensor carpi ulnaris (ECU), flexor carpi radialis (FCR) and flexor carpi ulnaris (FCU). The musculoskeletal model in physiological view is illustrated in Fig.1. FCR and FCU contribute to flexion, ECRB, ECRL and ECU contribute to extension, FCR, ECRL and ECRB contribute to radial deviation, FCU and ECU contribute to ulnar deviation. The contribution of these five muscles to pronation-supination (PS) is very small. Actually, PS motion occurs proximally to the wrist and it is often not considered as a pure wrist motion [8]. Obviously, the muscles are working in a coupled way to generate the 3D wrist motions. Even only one muscle receives abnormal tremulous neural drives, the wrist may exhibit tremor disorder in three directions. In order to implement the experiment easily, we regard the ECRB and ECRL as one muscle because the location of the two muscles is so close that the surface FES technique cannot stimulate each muscle separately. In mathematical representation, the musculoskeletal model has two parts, i.e.

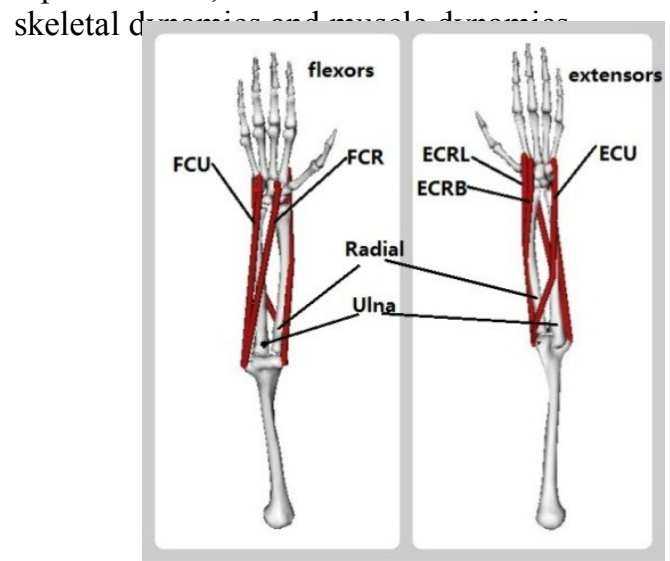

Fig.1 Simplified musculoskeletal model of wrist joint from the OpenSim

$$
\text { software [9]. }
$$

\section{A. Skeletal Dynamics}

For the 1-DOF wrist joint dynamics, for example the wrist radial-ulnar deviation moment, we can regard the joint as a single pendulum,

$$
T_{t}-T_{p}+T_{g}=J_{z} \ddot{\theta}
$$

where $T_{t}$ is the moment muscle generates through active electrical stimulation; $T_{p}$ is the internal passive moment; $T_{g}$ is the moment due to gravity. We assume gravity produces a positive angle and that the forearm is always perpendicular with respect to gravity and no pronation-supination occurs.

$$
T_{g}=m g d \cos \theta
$$


where $m$ and $d$ are respectively the hand mass and the distance between the hand center of mass and the wrist joint; $g$ is the mean local gravity acceleration.

For the 3-DoF wrist joint dynamics, that is wrist flexion-extension (FE), radial-ulnar deviation (RUD), and pronation-supination (PS). FE is parallel and RUD is vertical to the ground. The segment dynamics can be written as

$\left\{\begin{array}{c}T_{1}-T_{p 1}=J_{1} \ddot{\theta_{1}} \quad \text { Flexion }(+)-\operatorname{extension}(-) ; \\ T_{2}+T_{g}-T_{p 2}=J_{2} \ddot{\theta_{2}} \text { Radial }(+)-\operatorname{Ulnar}(-) \text { deviation; } \\ T_{3}-T_{p 3}=J_{3} \ddot{\theta}_{3} \quad \text { Pronation }(+)-\operatorname{supination}(-) ;\end{array}\right.$ where $\theta_{1}, \theta_{2}, \theta_{3}$ are the joint angles defined in FE, RUD and PS directions; $T_{1}, T_{2}, T_{3}$ are respectively the muscle moment in FE, RUD, and PS directions; $J_{1}, J_{2}, J_{3}$ are respectively the wrist inertia of the three directions. The coupling between the DoFs is not considered, i.e, coupling stiffness, damping and inertial interaction [8].

The values of related parameters in skeletal dynamics are referred to Table 1 [10]. The subject weight and height were used to calculate corresponding parameters.

Table 1. Some parameters of a specific subject whose weight is $73.0 \mathrm{~kg}$, height is $1.741 \mathrm{~m}$

\begin{tabular}{|c|c|c|c|c|}
\hline$J_{1}\left(\mathrm{gm}^{2}\right)$ & $J_{2}\left(\mathrm{gm}^{2}\right)$ & $J_{3}\left(\mathrm{gm}^{2}\right)$ & $m(\mathrm{~g})$ & $d(\mathrm{~cm})$ \\
\hline 1.305 & 0.871 & 1.784 & 445.3 & 8.6 \\
\hline
\end{tabular}

\section{B. Muscle Model}

The muscle model is developed from the classic Hill-type muscle model [2, 4], which consists of activation and contraction dynamics. The muscle model is illustrated in Fig. 2.

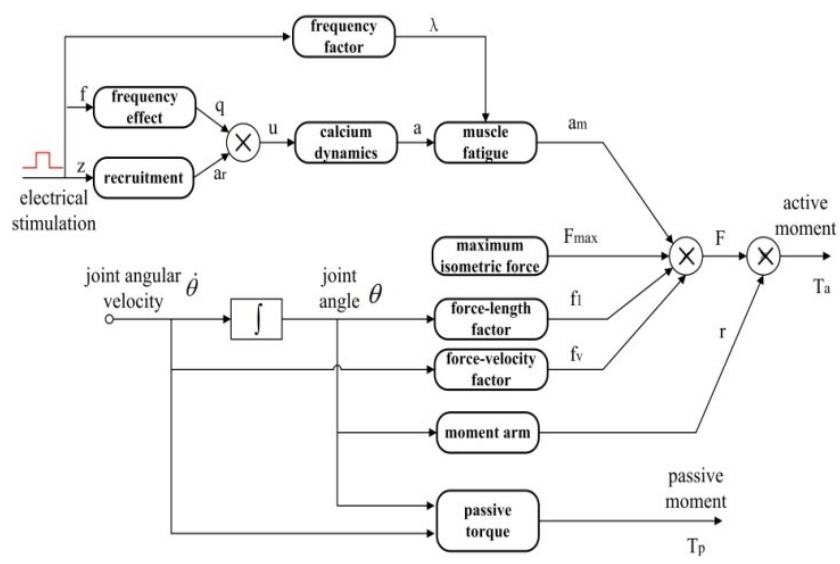

Fig. 2 Block diagram of muscle model.

\section{B.1 Muscle Contraction Dynamics}

The force which is generated by muscle through the stimulation is called the active force.

$$
\mathrm{F}=F_{\text {max }} \times f_{l} \times f_{v} \times a_{m}
$$

where $F_{\text {max }}$ is the muscle maximum isometric force; $f_{l}$ is the force-length factor; $f_{v}$ is the force-velocity factor; $a_{m}$ is the muscle activation with fatigue, $a_{m}=a p$, where $p$ is the muscle fatigue factor which is introduced later.
The active moment $T_{t}$ for 1-DoF wrist joint in flexion-extension is:

$$
T_{t}=F_{\text {flex }} \times r_{\text {flex }}-F_{\text {exten }} \times r_{\text {exten }}
$$

For 3-DoFs wrist joint:

$$
\left[\begin{array}{l}
T_{1} \\
T_{2} \\
T_{3}
\end{array}\right]=M\left[\begin{array}{l}
F_{E C R B} \\
F_{E C U} \\
F_{F C R} \\
F_{F C U}
\end{array}\right]
$$

where the moment arm matrix $M$ is obtained from the OpenSim software.

$$
\mathrm{M}=\left[\begin{array}{cccc}
-0.016 & -0.006 & 0.015 & 0.015 \\
-0.014 & 0.025 & -0.007 & 0.018 \\
0.0025 & 0.0002 & 0.0042 & 0.0024
\end{array}\right] \text {. }
$$

The muscle length change affects much active force strongly. A Gaussian-like function $f_{l}$ is used to describe the relationship between the muscle force and muscle fiber length.

$$
f_{l}=\exp \left[-\left(\frac{\bar{l}-1}{\varepsilon}\right)^{2}\right]
$$

where $\bar{l}$ is the normalized muscle length with respect to the optimal muscle length: $\bar{l}=l_{m} / l_{\text {opt }} ; l_{m}=l_{\text {opt }}+r \theta ; r$ is the moment arm and $\theta$ is the wrist angle. Then $\Delta l_{m}=$ $l_{m}-l_{\text {opt }}=r \theta$, thus it can be rewritten as

$$
f_{l}=\exp \left[-\left(\frac{r \theta}{l_{o p t}}\right)^{2}\right]
$$

If the wrist moves in 3 directions, the change of the four muscle lengths can be obtained as

$$
\left[\begin{array}{l}
l_{1} \\
l_{2} \\
l_{3} \\
l_{4}
\end{array}\right]=M^{T}\left(\begin{array}{l}
\theta_{1} \\
\theta_{2} \\
\theta_{3}
\end{array}\right)+\left(\begin{array}{l}
l_{10} \\
l_{20} \\
l_{30} \\
l_{40}
\end{array}\right)
$$

As we can get the length change of four muscles respect to 3 directions, then $f_{l}$ of each muscle can be computed.

The force-velocity $f_{v}$ can be used to model the effect between the muscle shortening/lengthening velocity and muscle force [4].

$$
f_{v}=0.54 \arctan (5.69 \bar{v}+0.51)+0.745
$$

where $\bar{v}$ is the normalized muscle velocity with respect to the maximum contraction (shortening) velocity $v_{\max }$ of the muscle: $\bar{v}=v_{m} / v_{\max }=r \dot{\theta} / v_{\max }$.

Similar with length of muscle, we can get the velocity $v_{m}$ of each muscle if wrist joint moves in three directions.

$$
\left[\begin{array}{l}
v_{m 1} \\
v_{m 2} \\
v_{m 3} \\
v_{m 4}
\end{array}\right]=M^{T}\left(\begin{array}{c}
\dot{\theta_{1}} \\
\dot{\theta_{2}} \\
\dot{\theta_{3}}
\end{array}\right)
$$

Table 2. The parameters of wrist muscles

\begin{tabular}{|c|c|c|c|}
\hline $\begin{array}{c}\text { Wrist } \\
\text { muscles }\end{array}$ & $F_{\text {max }}(\mathrm{N})$ & $l_{\text {opt }}(\mathrm{m})$ & $v_{\max }(\mathrm{m} / \mathrm{s})$ \\
\hline ECRB & 122.9 & 0.0585 & 10 \\
\hline ECU & 117 & 0.0622 & 10 \\
\hline FCR & 59.7 & 0.0628 & 10 \\
\hline FCU & 102.6 & 0.0509 & 10 \\
\hline
\end{tabular}


The values of related parameters in contraction dynamics are adopted from OpenSim software as shown in Table 2 [9].

The passive moment in the model can be given by [11].

$$
T_{p}=K_{p} \theta+B_{p} \dot{\theta}+M_{p n}
$$

where $M_{p n}$ is a nonlinear term given by

$$
M_{p n}=k_{2} \frac{\exp \left(k_{1}\left(\theta / k_{3}\right)\right)}{\exp \left(k_{1}\right)}-k_{2} \frac{\exp \left(k_{1}\left(-\theta / k_{3}\right)\right)}{\exp \left(k_{1}\right)}
$$

where $K_{p}, B_{p}, k_{1}, k_{2}, k_{3}$ are constant parameters.

For flexion-extension:

$K_{p}=0.4 ; B_{p}=0.3 ; k_{1}=6 ; k_{2}=0.3 ; k_{3}=0.8$;

For radial-ulnar deviation:

$K_{p}=0.3 ; B_{p}=0.3 ; k_{1}=6 ; k_{2}=0.1 ; k_{3}=0.3$;

For pronation-supination:

$K_{p}=0.4 ; B_{p}=0.3 ; k_{1}=6 ; k_{2}=0.03 ; k_{3}=1 ;$

\section{B.2 Muscle Activation Dynamics}

When a muscle is stimulated by electrical pulses, there is a dynamic process for the muscle to generate the force. This electrical characteristic is called as activation dynamics.

Muscle Recruitment Curve: It can be modeled by a piece-wise function with three values: a threshold pulse width (recruitment dead band) $P W_{d}$, a saturation pulse width $P W_{s}$. The pulse width of the electrical pulse is defined as $z$, the normalized muscle recruitment curve $a_{r}$ can be described in the following way:

$$
a_{r}=\left\{\begin{array}{cl}
0 & \text { for } z \leq P W_{d} \\
\frac{z-P W_{d}}{P W_{s}-P W_{d}} & \text { for } P W_{d} \leq z \leq P W_{s} \\
1 & \text { for } z>P W_{s}
\end{array}\right.
$$

Frequency Characteristics: When the frequency of stimulation pulse varies, it also affects the force produced by the muscle. This force-frequency characteristic can be defined by

$$
\mathrm{q}=\frac{(0.1 f)^{2}}{1+(0.1 f)^{2}}
$$

where $\mathrm{q}$ is the characteristic factor of the stimulation frequency $f$.

Calcium Dynamics: It can be modeled as a first order differential equation

$$
\dot{a}=\frac{1}{\tau_{a c}}\left(u^{2}-u a\right)+\frac{1}{\tau_{d a}}(u-a)
$$

where $a$ is the muscle activation without fatigue, $\mathrm{u}=a_{r} q$, $\tau_{a c}$ is activation time constant and $\tau_{d a}$ is de-activation time constant.

Muscle Fatigue: It depends on the activation level $a$ and frequency $f$ of the stimulation.

$$
\begin{gathered}
\dot{p}=\frac{\left(p_{\text {min }}-\mathrm{p}\right) a \lambda}{\tau_{f a t}}+\frac{(1-p)(1-a \lambda)}{\tau_{\text {rec }}} \\
\lambda=1-\beta+\beta\left(\frac{f}{100}\right)^{2}
\end{gathered}
$$

where $\mathrm{p}$ is the fatigue factor, $\tau_{\text {fat }}$ is the fatigue time constant, $\tau_{r e c}$ is the recovery time constant, $p_{\min }$ is the minimum fitness, $\lambda$ is the frequency factor on fatigue, and $\beta$ is the shaping factor.
The values of related parameters in activation dynamics are referred to [4] and listed in Table 3.

Table 3. Parameters of the wrist joint model

\begin{tabular}{|c|c|c|c|c|c|}
\hline$\tau_{a c}(\mathrm{~ms})$ & $\tau_{d a}(\mathrm{~ms})$ & $\varepsilon$ & $\beta$ & $P W_{d}(\mu \mathrm{s})$ & $P W_{s}(\mu \mathrm{s})$ \\
\hline 40 & 70 & 0.4 & 0.6 & 100 & 500 \\
\hline
\end{tabular}

\section{Simulation StUdy}

The simulation study is carried out in Matlab/Simulink environment. The wrist tremor can be achieved when the related muscles are activated by tremulous stimulation. In real-world patients, the tremulous stimulation can be measured and represented by EMG signals. Similar with EMG, artificial electrical pulses can be applied to the muscle via FES technique to generate the artificial tremor. Three variables of electrical pulse can be regulated, i.e. pulse width, pulse frequency, and pulse amplitude. Pulse width $(\mathrm{PW})$ is the only controlled variable here. The other two variables are set as constants, pulse frequency is $20 \mathrm{~Hz}$ and pulse amplitude is $17 \mathrm{~mA}$.
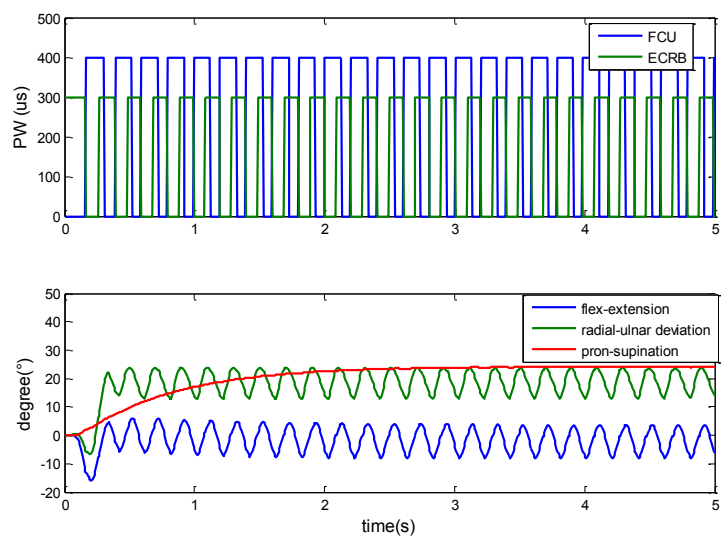

Fig. 3 Upper plot shows intensity (PW) of electrical pulse applied to FCU and ECRB. Lower plot shows the wrist joint angle in three directions.

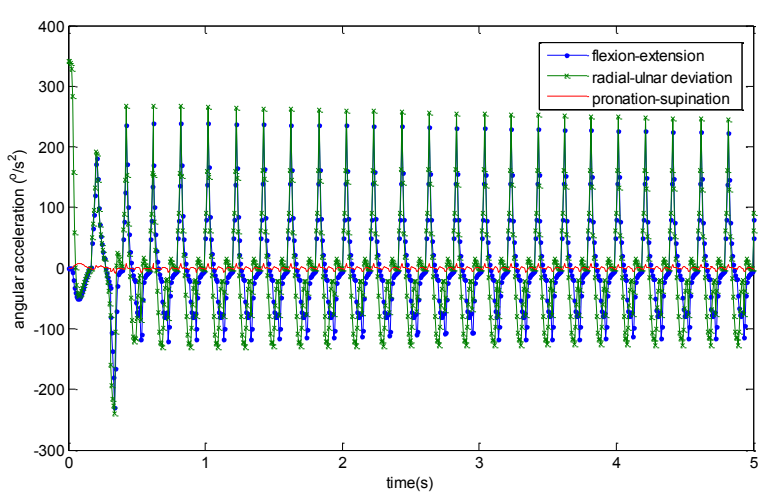

Fig. 4 Angular acceleration of wrist joint in three directions.

According to the EMG pattern and intensity acquired from patients [4], the electrical stimulation to ECRB $(\mathrm{PW}=300 \mu \mathrm{s})$ and FCU $(\mathrm{PW}=400 \mu \mathrm{s})$ is provided. The stimulation frequency is $5 \mathrm{~Hz}$. Note the stimulation frequency is different from the pulse frequency. We can get 
the wrist angles as shown in Fig.3, the result matches the fact that ECRB can generate negative flexion angle, negative deviation angle and positive pronation angle when it is activated; while FCU can produce positive flexion angle, positive deviation angle and positive pronation angle. The angular acceleration in each direction is obtained in simulation study as shown in Fig.4.

\section{EXPERIMENTAL STUDY}

The experimental equipments include FES stimulator (Compex Motion II, Switzerland), goniometer (Biometrics, UK) and accelerometer (Delsys Trigno Wireless System, USA). During experiments, the subjects naturally held their arm on a horizontal level. The hand was open, and the RUD direction of wrist joint was perpendicular to the ground. In comparison with the simulation study, the muscles ECRB and FCU were stimulated to generate the artificial tremor. The goniometer measured the wrist joint angle of FE and RUD. The accelerometer measured the acceleration of wrist joint in three directions. Fig. 5 shows the measurement sensors and the location of FES electrodes on the subject. In this case, the surface electrodes are attached to the forearm, and through the electrodes, electrical pulses can stimulate the muscles to generate wrist flexion-extension and radial-ulnar deviation.

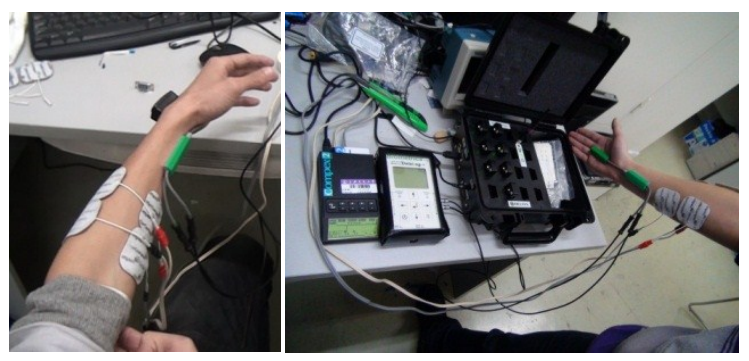

Fig. 5 Sensors and FES electrodes on the subject for tremor generation.

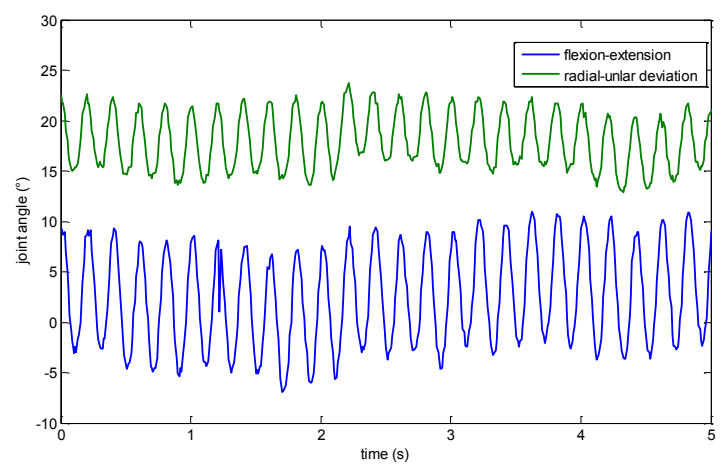

Fig. 6 Wrist joint angle of the subject measured by goniometer under FES.

The parameters of stimulation are the same as that in simulation study. The data of joint angles are shown in Fig.6, which qualitatively match the simulation results as shown in Fig.3. The acceleration of wrist joints is shown in Fig.7, and the unit is scaled by acceleration of gravity $(g)$. Translation does not occur in the wrist joint [8], and the corresponding data in X-direction may be ignored. The difference in quantity between simulation results and experimental results may attribute to: 1) The values of parameters in the musculoskeletal model are adopted form literatures, which are different from those of the subjects in real experiments; 2) the current model is still a simplified version of $3 \mathrm{D}$ wrist joint, there is a certain gap between the model and the real one. For example, the interaction dynamics among the three DoFs is not considered. However, it does not impair the significance of the current work, since there is not a general musculoskeletal model that can match every person in the world. Credibility and tractability of the model should be compromised.

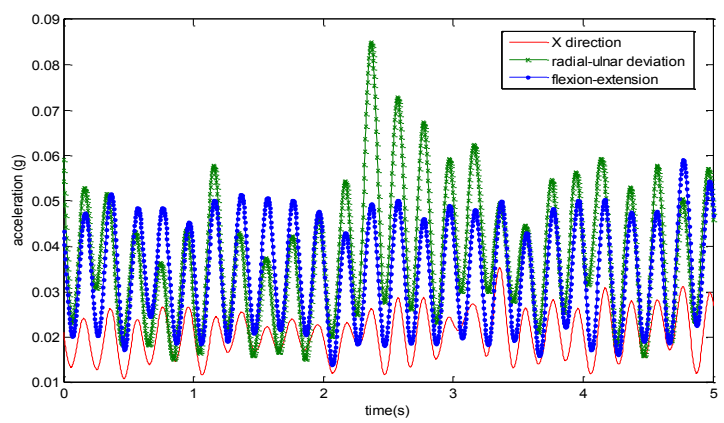

Fig. 7 Wrist joint acceleration of the subject measured by accelerometer under tremor generation using FES.

\section{CONCLUSION}

The 3-dimentional musculoskeletal model of wrist joint with multiple muscles to study tremor is developed in our work. The tremor simulation based on the model is performed in Matlab/Simulink environment. The artificial tremor generation is conducted on healthy subjects via FES to verify the model performance. Results have shown the model can generate similar tremor with that in experiment. It implies that this model may have promising application that considers all DOFs of wrist joints in tremor analysis in future.

\section{REFERENCES}

[1] R.J. Elble and W.C. Koller, Tremor, The Johns Hopkins University Press, 1990

[2] D.G. Zhang, P. Poignet, A.P.L. Bo, and W. T. Ang, "Exploring peripheral mechanism of tremor on neuromusculoskeletal model: A general simulation study," IEEE Trans. Biomedical Engineering, vol.56, pp.2359-2369, 2009.

[3] I. Fukumoto, "Computer simulation of Parkinsonian tremor," J. Biomed. Eng., vol. 8, pp. 49-55, 1986.

[4] D.G. Zhang, P. Poignet, F. Widjaja, and W.T. Ang, "Neural oscillator based control for pathological tremor suppression via functional electrical stimulation," Control Engineering Practice, vol.19, pp.74-88, 2011.

[5] A.P.L. Bo, P. Poignet, D.G. Zhang, and W.T. Ang, "FES-controlled co-contraction strategies for pathological tremor compensation," in Proceedings of IEEE Conf. IROS, pp. 1633-1638, 2009.

[6] R. Ruwadi, P. Poignet, M.L. Han, and W.T. Ang, "Single-input single-output EMG-based musculoskeletal modeling for hand tremulous motion," in Proceedings of $3^{\text {rd }}$ IEEE Conf. BioRob, pp.924 - 929, 2010.

[7] J.L. Dideriksen, R.M. Enoka, and D. Farina, "A model of the surface electromyogram in pathological tremor," IEEE Trans. Biomedical Engineering, vol.58, pp.2178-2185, 2011.

[8] S.K. Charles and N. Hogan, "Dynamics of wrist rotation," $J$. Biomechanics, vol.44, pp.614-621, 2011.

[9] https://simtk.org/project/xml/downloads.xml?group id=91

[10] Paolo de Leva. "Adjustments to Zatsiorsky-seluyanov's segment inertia parameters" J. Biomechanics. vol.29, no.9, pp.1223-1230, 1996. [11] T.Edrich, R.Riener, and J. Quintern, "Analysis of passive elastic joint moments of the lower extremities in paraplegics and normal controls," IEEE Trans. Biomed. Eng, vol.47, pp.1058-1065, Aug.2000. 